\title{
A Study of Adverse Cutaneous Drug Reactions Due to Nsaids at a Rural Based Tertiary Care Centre, Gujarat
}

\author{
Rita Vora ${ }^{{ }^{*}}$, Singhal Rochit $\mathrm{R}^{2}$, Patel Trusha $\mathbf{M}^{3}$ and Modasia Khushboo $\mathbf{H}^{4}$ \\ Department of Dermatology \& Venereology, Pramukhswami Medical College, Karamsad, Gujarat, India
}

Received: January 06, 2018; Accepted: January 29, 2018; Published: May 25, 2018

*Corresponding author: Rita Vora, Department of Dermatology \& Venereology, Pramukhswami Medical College, Karamsad, Gujarat 388325, India, Tel: 9879290417; E-mail: ritavv@charutarhealth.org

\section{Abstract}

Objective: To find out various clinical patterns of ACDR (Adverse Cutaneous Drug Reaction) due to NSAIDS, the most common NSAIDS responsible for it.

Materials and method: The study was cross sectional and was carried out in the department of dermatology in a teaching institute at a rural based tertiary care centre of Gujarat from March-2010 to March 2017.

Results: A total of 288 patients enrolled in the study, among which 173 (60.07\%) were males and 115 (39.93\%) were females. Most common age group was 21-40 years. Most common presenting complaint was redness (which was generalized in some and localized in few) in $61(26.75 \%)$ patients followed by itching in $58(25.43 \%)$ patients. With most common diagnosis of cutaneous ADR were urticaria 97(33.6\%), angioedema 45(15.6\%), FDR (Fixed Drug Reaction) 17(5.9\%), DRESS (Drug Reaction with Eosinophilia and Systemic Symptoms) 11(3.8\%), SJS(Stevenson Johnson Syndrome) $9(3.1 \%)$, TEN (Toxic Epidermal Necrolysis) $1(0.34 \%)$ and others $37(12.8 \%)$

Conclusion: Pharmacovigilance improves the recognition of ADRs and helps the medical professional to have safe practice.

Keywords: Adverse drug reaction, pharmacovigilance, non steroidal anti-inflammatory drug

Abbreviations: ACDR (Adverse Cutaneous Drug Reaction)

FDR (Fixed Drug Reaction

DRESS (Drug Reaction with Eosinophilia and Systemic Symptoms)

SJS (Stevenson Johnson Syndrome)

TEN (Toxic Epidermal Necrolysis)

OTC (Over the Counter)

\section{Introduction}

Cutaneous manifestations are common adverse drug reactions. Non Steroidalanti Inflammatory Drugs (NSAIDS) are not only one of the most commonly used drugs but also those which commonly cause Adverse Cutaneous Drug Reaction (ACDRs). NSAIDS can cause urticaria, angioedema, acneform eruption, SJS, TEN, vasculitis etc. It is very important and useful to have detailed knowledge regarding ACDRs due to NSAIDS. Cyclooxygenase (COX) inhibitors, commonly called Non steroidal Anti-Inflammatory Drugs (NSAIDs), such as ibuprofen, diclofenac, and naproxen, have anti-inflammatory and analgesic/ antipyretic properties across a wide range of dosing regimens. All NSAIDs are COX inhibitor that converts arachidonic acid to prostaglandins [1]. Main ADRs associated with NSAIDs are in gastro $i$ intestinal (GI), cardiovascular (CV), and renal sites [2]. Cutaneous manifestations are rare but serious.

\section{Material and Methods}

The study was carried out in the department of dermatology in a teaching institute at a rural based tertiary care centre of Gujarat from March-2010 to March 2017 after getting ethical approval from HREC of the institute. This was a cross sectional analytical study. The study included all the patients with symptoms and signs suggestive of adverse cutaneous drug reaction after intake of NSAIDS. Inclusion criteria: (1) Any age- group (2)Any gender (3) Any CADR due to NSAID drug (4) Gives written informed consent: Exclusion criteria (1) Any patient with more than one drug allergy (2) Patient refused to give written informed consent. All the data was recorded in a predesigned proforma with the consent of patients and analysis was done. An attention was paid to the drug history, temporal correlation with the drug, duration of the rash, appearance of signs and symptoms, morphology of the eruption, associated mucosal or systemic involvement and improvement of lesions on withdrawal of drug. In every case a detailed history was elicited and a thorough clinical examination was carried out. To establish the etiologic agent for a particular type of reaction, a diagnosis of ACDR was reached after exclusion of other aetiologies and similar disorders like reactions due to food, infections and environmental factors. If more than one drug was thought to be responsible, the most likely offending agent was noted and the impression was confirmed by subsidence of the rash on withdrawing the drug. Naranjos ADR causality was 
also used to evaluate CADRs [3]. HartwigsPreventilibility and severity scale was also utilized [4].

\section{Results}

A total of 288 patients enrolled in the study, among which $173(60.07 \%)$ were males and 115 (39.93\%) were females. Most common age group was 21-40 years both in males and females (Table 2). Most common presenting complaint was redness in 61 (26.75\%) patients followed by itching in $58(25.43 \%)$ patients. Latency period after taking of the drug and development of lesions was 3-5 days in 119(41.31\%) of patients and it was 5-7 days in $61(21.18 \%)$, It was $1-2$ day in $37(12.84 \%)$ patients (Table 1). In our study the difference between latency period of NSAIDs according to WHO-UMC score and Naranjo was not compared.265 (92.01\%) developed CADR to the prescribed drugs while rest 23(7.98\%) developed to self administered drugs. Route of administration was oral in $247(85.76 \%)$ patients and parenteral in $41(14.23 \%)$ patients. Past history of cutaneous drug reaction was seen in 69 (23.95\%) patients out of which past history of cutaneous drug reaction with same drug was seen in 31(10.76\%) patients and was not only due to NSAIDs. Family history of cutaneous drug reaction was seen in $15(5.2 \%)$ patients. Atopy or

\begin{tabular}{|c|c|c|}
\hline \multicolumn{2}{|l|}{ Table 1: Incubation period of ACDRs } \\
\hline Duration of appearance & No. of patients & $\mathbf{\%}$ \\
\hline Less than $1 \mathrm{hr}$ & 0 & 0 \\
\hline $1-6 \mathrm{hr}$ & 5 & 1.73 \\
\hline $6-24 \mathrm{hr}$ & 13 & 4.51 \\
\hline 1-2 day & 37 & 12.84 \\
\hline 3-5 days & 119 & 41.31 \\
\hline 5-7 day & 61 & 21.18 \\
\hline 1-2 weeks & 32 & 11.11 \\
\hline$>2$ weeks & 21 & 7.29 \\
\hline
\end{tabular}

Table 2: Age and sex distribution of patients of ACDR due to NSAIDS

\begin{tabular}{|c|c|c|c|}
\hline Age group & Males & Female & Total \\
\hline $0-20$ & 24 & 15 & 39 \\
\hline $21-40$ & 89 & 54 & 143 \\
\hline $41-60$ & 36 & 19 & 55 \\
\hline $61-80$ & 12 & 13 & 25 \\
\hline$>80$ & 12 & 14 & 26 \\
\hline Total & 173 & 115 & 288 \\
\hline
\end{tabular}

Table 3: Various patterns of drug reactions owing to various drugs

\begin{tabular}{|c|c|c|c|c|c|c|c|c|c|c|c|}
\hline & MDR & FDR & Urticaria & Angioedema & $\begin{array}{l}\text { Acneform } \\
\text { eruptions }\end{array}$ & SJS & TEN & Ex ds & Vasculitis & DRESS & Total \\
\hline Aspirin & 5 & 1 & 16 & 4 & 2 & 1 & 0 & 6 & 0 & 1 & $36(12.5 \%)$ \\
\hline Paracetamol & 5 & 3 & 13 & 7 & 3 & 2 & 0 & 4 & 0 & 1 & $38(13.2 \%)$ \\
\hline Diclofenac & 6 & 2 & 14 & 5 & 4 & 1 & 1 & 5 & 0 & 0 & $38(13.2 \%)$ \\
\hline Ibuprofen & 5 & 3 & 13 & 7 & 3 & 2 & 0 & 3 & 0 & 3 & $39(13.5 \%)$ \\
\hline Indomethacin & 8 & 3 & 13 & 7 & 3 & 2 & 0 & 4 & 0 & 0 & $40(13.9 \%)$ \\
\hline Etoricoxib & 1 & 2 & 4 & 3 & 4 & 0 & 0 & 3 & 1 & 2 & $20(7 \%)$ \\
\hline Naproxen & 5 & 1 & 15 & 5 & 5 & 1 & 0 & 5 & 0 & 0 & $37(12.9 \%)$ \\
\hline Nimesutide & 1 & 1 & 5 & 4 & 3 & 0 & 0 & 5 & 0 & 2 & $21(7.3 \%)$ \\
\hline Parecoxib & 1 & 1 & 4 & 3 & 3 & 0 & 0 & 5 & 0 & 2 & $19(6.5 \%)$ \\
\hline Total & 37 & 17 & 97 & 45 & 30 & 9 & 1 & 40 & 1 & 11 & 288 \\
\hline
\end{tabular}

allergic tendency was seen in $23(7.99 \%)$ patients. 150 patients $(52.08 \%)$ had body surface area involvement less than $25 \%, 77$ (26.73\%) patients had involvement between $25-50 \%$ while 46 (15.97\%) patients had involvement between 51-75\%. Only 15 patients $(5.20 \%)$ had body surface area more than $75 \%$. Severity of reaction according to Hartwigs Adverse Drug Reaction (ADR) severity assessment, was mild in 221 (76.73\%), moderate in 58 (20.14\%), severe in 9 (3.12\%) patients. Naranjo ADR probability scale, was definite in $34(11.80 \%)$, probable in $201(69.79 \%)$, possible in 53(18.40\%) and doubtful in 0 cases. Most common mucosal surface involved was oral mucosa in 20 (16.7\%) patients followed by genital mucosa in $19(15.8 \%)$ patients, conjunctival in $7(5.8 \%)$ patients, anal mucosa in $5(4.2 \%)$ patients. Out of 288 patients, $40(13.9 \%)$ were due to indomethacin,30(13.5\%) ibuprofen,38(13.2\%) paracetamol,38(13.2\%)diclofenac and $37(12.9 \%)$ naproxen. Most common type of ACDR due to NSAIDS is urticaria $97(33.6 \%)$, angioedema $45(15.6 \%)$, exfoliative dermatitis 40(13.8\%), acneform eruptions 30(10.4\%), FDR $17(5.9 \%)$, DRESS $11(3.8 \%)$, SJS $9(3.1 \%)$, vasculitis $1(0.34 \%)$, TEN $1(0.34 \%)$ and others $37(12.8 \%)$.

\section{Discussion}

ADR is defined as "an appreciably harmful or unpleasant reaction, resulting from an intervention related to the use of a medicinal product, which predicts hazard from future administration and warrants prevention or specific treatment, or alteration of the dosage regimen, or withdrawal of the product" [5]. Adverse drug reactions are classified into six types (with mnemonics): dose-related (Augmented), non-doserelated (Bizarre), dose-related and time-related (Chronic), time-related (Delayed), withdrawal (End of use), and failure of therapy (Failure) [6]. Drug reactions can be classified into 


\begin{tabular}{|c|c|}
\hline \multicolumn{2}{|c|}{ Table 4 : Various ACDR assessment scores } \\
\hline WHO-UMC Score & No of patients (\%) \\
\hline Casuality term & $30(10.41 \%)$ \\
\hline Certain & $160(55.55 \%)$ \\
\hline Propable & $55(19.09 \%)$ \\
\hline Possible & $18(6.25 \%)$ \\
\hline Unlikely & $13(4.51 \%)$ \\
\hline Conditioned & $12(4.17 \%)$ \\
\hline Unclassifiable & \\
\hline Naranjo ADR probability scale & $34(11.80 \%)$ \\
\hline Definite & $201(69.79 \%)$ \\
\hline Probable & $53(18.40 \%)$ \\
\hline Possible & 0 \\
\hline Doubtful & $111(38.54 \%)$ \\
\hline Hartwigs severity assessment scale & $110(38.19 \%)$ \\
\hline Level 1 (Mild) & $42(14.58 \%)$ \\
\hline Level 2 (Mild) & $16(5.55 \%)$ \\
\hline Level 3 (Moderate) & $7(2.43 \%)$ \\
\hline Level 4 (Moderate) & $2(0.69 \%)$ \\
\hline Level 5 (Severe) & $0(0 \%)$ \\
\hline Level 6 (Severe) & \\
\hline Level 7 (Severe) & \\
\hline
\end{tabular}

immunologic and nonimmunelogicetiologies. The majority $(75$ to 80 percent) of adverse drug reactions are caused by predictable, nonimmunologic effects.1. The remaining 20 to 25 percent of adverse drug events are caused by unpredictable effects that may or may not be immune mediated [7]. Non steroidal anti inflammatory drugs is a class of analgesic medication that reduces pain, fever and inflammation. Non Steroidal AntiInflammatory Drugs (NSAIDs) have a long history of its use as both prescription and over-the counter (OTC) analgesics/ antipyretics. All NSAIDs inhibit COX, an enzyme that converts arachidonic acid to prostaglandins. In the process, prostaglandin $\mathrm{H} 2$ is converted to five primary prosta $\neg$ glandins, including thromboxane A2 (which stimulates platelet aggregation and blood clot formation) in plate 7 lets and prostacyclin (a vasodilator that inhibits platelet aggregation) in the endothelium [8]. Conventional NSAIDs are nonselective, which bind and inhibit both the isoforms, but cyclooxygenase-1 (COX-1) is inhibited more avidly than cyclooxygenase-2 (COX-2) [9]. Inhibition of COX-1 is responsible for the side effects and that of COX-2 for therapeutic effects. This has resulted in the introduction of the COX-2 selective drugs [10]. As all the other drugs, NSAIDs also have its own adverse drug reactions affecting various systems of the body. The specific risk factors for NSAID ADR are older age, a history of gastro-duodenal ulcer, dyspepsia, concomitant use of medications such as corticosteroids and anticoagulants, high dosage use of multiple NSAIDs and the presence of other chronic co-morbidities [11]. As recommended by the European Medicine Agency (EMA), NSAIDs should be prescribed at the lowest effective dose and for the shortest time necessary to control symptoms [12]. ADRs associated with NSAIDs primarily manifest in gastro $\neg$ intestinal (GI), cardiovascular (CV), and renal sites [13]. The most frequent serious ADRs reported with the selected oral NSAIDs are cutaneous, followed by gastrointestinal, hepatic and renal events [14]. In a study of 100 orthopedicpatients of adverse drug reactions, Total 3 patients had cutaneous adverse drug reactions which included urticaria, itching and redness of the skin [15]. A study of adverse drug reactions was done in pediatric age group including 754 cases over the period of 5 years.. Among this 25 patients showed adverse drug reactions due to NSAIDs and 10 showed cutaneous adverse drug reaction [16]. To evaluate the safety profile of oral Non Steroidal Anti-Inflammatory Drugs (NSAIDs) available in France a study was conducted from 2002 to 2006. A total of 42389 ADRs were reported and among them cutaneous drug reactions were observed in 6052 patients [14]. Patel et al has done a study of cutaneous reactions due to various drugs in 200 patients among which NSAIDs were the most common drug, 11 showed morbiliform rash, 20 showed urticaria and angioedema and 19 showed fixed drug reaction [17].

In our study out of 288 patients of ACDR FDR was seen in $17(5.9 \%)$ and Urticaria in $97(33.6 \%)$ while in Patel et al cases with FDR were 61(30.5\%) and Urticaria were 37(18.5\%) thappa et al presented ACDR in total $12 \%$ cases due to Nsaids [18].

After extensive literature search we could find the research which included all the adverse drug reactions due to NSAIDs but not many having specific information regarding cutaneous manifestations.

\section{Conclusion}

Nsaids are one of the most commonly used OTC (Over the Counter) drugs. The patient's education regarding using the lowest effective dose of OTC NSAIDs for the shortest required duration is vital in balancing efficacy and safety is to be provided by health care professionals. Pharmacovigilance improves recognition of ADRs by the medical professionals. It allows the treating physician to identify the ADR associated with drugs, in particular, with the ones considered relatively safe and with those commonly prescribed by the medical and non-health professionals. Pharmacovigilance is an important tool for the treating physician to develop safe medical practice.

\section{References}

1. Rao P, Knaus EE. Evolution of nonsteroidal anti-inflammatory drugs (NSAIDs): cyclooxygenase (COX) inhibition and beyond. J Pharm Pharm Sci. 2008;11(2):81s-110s.

2. Helin-Salmivaara A, Saarelainen S, Gronroos JM, Vesalainen R, Klaukka T, Huupponen R. Risk of upper gastrointestinal events with the use of various NSAIDs: a case-control study in a general population. Scand J Gastroenterol. 2007;42(8):923-932.

3. Naranjo CA, Busto U, Sellars EM, Sandor P, Ruiz I, Roberts EA, et al. Method for Estimating the Probability of Adverse Drug Reactions. Clin Pharmacol Ther. 1981;30(2):239-245.

4. Hartwig SC, Siegel J, Schneider PJ. Preventability and Severity Assessment in Reporting Adverse Drug reactions. Am J Hosp Pharm. 1992;49(9):2229-2232. 
5. Nayak S, Acharjya B. Adverse cutaneous drug reaction. Indian J Dermatol. 2008;53(1):2-8. doi: 10.4103/0019-5154.39732

6. Edwards IR, Aronson JK. Adverse drug reactions: definitions, diagnosis, and management. Lancet. 2000;356(9237):1255 - 1259.

7. Executive summary of disease management of drug hypersensitivity: a practice parameter. Joint Task Force on Practice Parameters, the American Academy of Allergy, Asthma and Immunology, the American Academy of Allergy, Asthma and Immunology, and the Joint Council of Allergy, Asthma and Immunology. Ann Allergy Asthma Immunol. 1999; 83(6 Pt 3):665-700.

8. Rao P, Knaus EE. Evolution of nonsteroidal anti-inflammatory drugs (NSAIDs): cyclooxygenase (COX) inhibition and beyond. J Pharm Pharm Sci. 2008;11(2):81s-110s.

9. Dhikav V, Singh S, Anand KS. Newer non-steroidal anti-inflammatory drugs: A review of their therapeutic potential and adverse drug reactions. JIACM. 2002;3(4):332-338.

10. Lipsy P. The role of cyclooxygenase-2-specific inhibitors in clinical practice. Am J Med 2001;110:1-5.

11. Fry RB, Ray MN, Cobangn DJ, Weissman NW, Kiefe CI, Shewchuk RM, et al. Racial / ethnic disparities in patient - reported non-steroidal anti-inflammatory drug (NSAIDs) risk awareness, patient - doctor NSAID risk communication and NSAID risk behavior. Arthritis Rheum. 2007;57(8):1539-1545.
12. European Medicine Agency EMA. General reccomendation about NSAIDs. 2011. Available from: http://www.ema.europa.eu/docs/en_ GB/document_library/Press_release/2009/11/WC500014477.pdf

13. Nicholas M,CharlesP,Paul B. Adverse drug reactions and drug-drug interactions with over-the-counter NSAIDs. Ther Clin Risk Manag. 2015;11:1061-1075. doi: 10.2147/TCRM.S79135

14. Maryse L, Sabrina G,Jean-L. Adverse drug reactions associated with the use of NSAIDs: a case/noncase analysis of spontaneous reports from the French pharmacovigilance database 2002-2006. Fundam Clin Pharmacol. 2013;27(2):223-230. doi: 10.1111/j.14728206.2011.00991.x

15. Alpa G, Miti S. Adverse drug reactions of nonsteroidal antiinflammatory drugs in orthopaedic patients. J Pharmacol Pharmacother. 2011;2(1):26-29. doi: 10.4103/0976-500X.77104

16. ThirzaT, NoelC, Sean B. Adverse drug reactions to nonsteroidal anti-inflammatory drugs, COX-2 inhibitors and paracetamol in a paediatric hospital. Br J Clin Pharmacol. 2005;59(6):718-723. doi: 10.1111/j.1365-2125.2005.02444.x

17. Patel Raksha M, Marfatia YS. Clinical study of cutaneous drug eruption in 200 patients. Indian J Dermatol Venereol Leprol. 2008;74(1):80.

18. Pudukadan D, Thappa DM. Adverse cutaneous drug reactions: Clinical pattern and causative agents in a tertiary care center in South India. Indian J Dermatol Venereol Leprol. 2004;70(1):20-24. 